\title{
SVM-based Tool to Detect Patients with Multiple Sclerosis Using a Commercial EMG Sensor
}

\author{
Konstantin Akhmadeev*, Aya Houssein*, Saïd Moussaoui*, \\ Einar A. Høgestøl' ${ }^{\dagger}$, Hanne F. Harbo ${ }^{\dagger}$, Steffan D. Bos-Haugen ${ }^{\ddagger}$, \\ Jennifer Graves ${ }^{\S}$, David Laplaud $₫$, Pierre-Antoine Gourraud $₫$ \\ *Laboratory of Digital Sciences of Nantes, Nantes, France, Email: konstantin.akhmadeev@1s2n.fr \\ $\dagger$ Department of Neurology, Institute of Clinical Medicine, University of Oslo, Oslo, Norway \\ $\ddagger$ Department of Neurology, Oslo University Hospital, Oslo, Norway

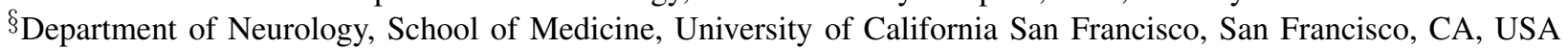 \\ ITransplantation and Immunology Research Centre, UMR1064, INSERM, University of Nantes, Nantes, France
}

\begin{abstract}
Multiple sclerosis (MS) is a major auto-immune disease that is the leading cause of non-traumatic impairment of the central nervous system (CNS) in young adults. Successful treatment of MS patients depends on accurate tools for both the MS diagnosis and the disability progression. The neurological examination is an expert procedure which aims to accurately describe the function of the nervous system, but despite the complexity of the neurological examination it still lacks accuracy. This study explores the capabilities of applying a commercial electromyographic and inertial sensor (MYO Armband by Thalmic Labs Inc.) coupled with a multichannel signal processing tool to standard neurological examination. In this pilot study, a dataset of electromyographic signals from 71 individuals (31 MS patients and 40 healthy controls) was acquired during neurological examination routine. Temporal and spectral features of the signals were extracted in order to train and validate a classification model. Finally, a Support Vector Machine classifier was obtained giving AUROC $=0.94,95 \% \mathrm{CI}=[0.88,0.99]$ and verified using five-fold cross-validation. We propose a set of signal descriptors that correlate with objective components of the neurological examination. The proposed signal acquisition and processing technique, being easy to integrate into the traditional neurological exam, may have high potential for aiding in diagnosing MS and quantifying its progression.
\end{abstract}

\section{INTRODUCTION}

Multiple sclerosis (MS) is a chronic debilitating neurological disorder that mainly affects young individuals aged between 20 and 40. As a cause of neurologic disability MS is second only to trauma, having its prevalence estimated at 2.5 million worldwide in 2014. The actual cause of MS is yet to be identified, but a complex interaction between genetic and environmental factors contributes to the risk. To date, there is no reliable method to predict MS onset or progression. Successful managing of the symptoms and attacks for MS patients highly depends on accurate and timely diagnosis as well as the possibility to measure disability progression.

Diagnostic criteria for multiple sclerosis include a number of clinical and paraclinical laboratory assessments [1], [2]: cerebrospinal fluid analysis, study of visual evoked potentials, electromyography analysis, neuroimaging and motor function tests. The latter involves various motor tasks to be accomplished by the subject: timed 25-foot walk [3], 9-hole peg test
[4], finger-to-nose test [5], heel-knee-shin test, finger tapping, foot tapping, etc.

The most common motor manifestations of MS are muscle fatigue, spasticity and tremor. Listed symptoms involve abnormal functioning of skeletal muscles and thus affect their activation patterns. In such cases, deviations may be revealed by analysis of limb trajectories and of involved muscles' electromyography (EMG). These measurements are proven to be efficient in different studies of MS progression [6]-[8]. Thus, an EMG recording along with the inertial measurement unit (IMU) data may aid to characterise presence and severity of MS.

Common MS diagnosis approaches, as those listed above, require specific equipment, procedures and clinical expertise. A lack of them may slow down or make the diagnosis impossible, which is a common case for unpopulated areas or developing countries. A possible way to overcome these difficulties is to apply a widespread cheap acquisition system, along with unified assessment protocol and automated decision-making. As such an acquisition system we propose the MYO armband (Figure 1) developed and commercialised by Thalmic Labs Inc [9]. It comprises eight EMG channels and an IMU giving acceleration, orientation and rotation speed measurements in three axes. This device is wireless, cheap, easy to use, actively supported by community and can be shipped to any location.

MYO armband's default software is capable of recognising five different hand gestures, based on EMG. Also, IMU sensor provides a pointer control. In academical studies, this device was applied in sign language gesture recognition [10] and prosthetic control [11], [12]. Typical signal processing pipeline in these applications consists of the following steps: windowing, feature extraction, feature projection and classification using machine learning techniques [13], [14]. Such an approach may also be effective in an application to MS diagnostics since there is no strictly defined model of how MS affects surface EMG signals or limb trajectories. Other reasons to use machine learning techniques in this case are the dimensionality of the data and the fact that measurements 


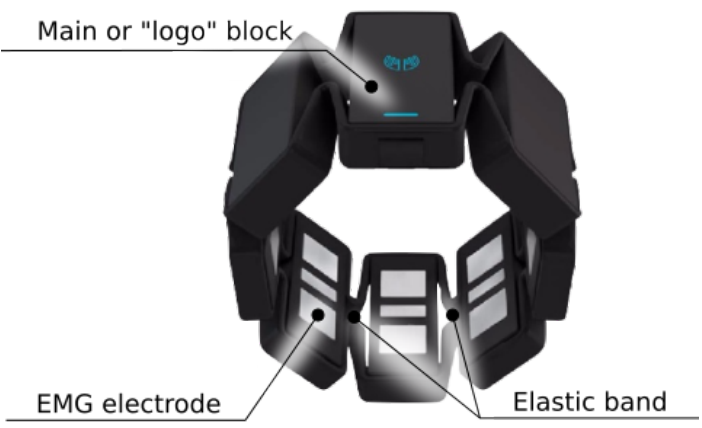

Fig. 1. MYO armband by Thalmic Labs Inc.

are to be made with two different kinds of sensors (EMG and IMU).

In the sequel, we present the entire processing pipeline including the data acquisition, signal preprocessing, dimensionality reduction and decision-making steps. At last, experimental results involving MS patients and healthy controls are discussed.

\section{Materials AND Methods}

This section contains description of our equipment, test protocol, data acquisition software and data processing approaches.

\section{A. Acquisition system}

As it was already mentioned, a proposed acquisition device was an armband consisting of eight blocks connected by an elastic rubber band. Each block has a differential surface EMG electrode on the side opposed to the skin. It also contains an integrated IMU providing acceleration and orientation data. The armband hardware itself performs filtering and sampling of the signals (EMG at $200 \mathrm{~Hz}$ and IMU at $50 \mathrm{~Hz}, 10 \mathrm{bit}$ quantisation) and may be connected wirelessly to a PC using Bluetooth Low Energy protocol.

A custom acquisition software with graphical user interface (figure 3) was developed to conduct the acquisition. It handled several major tasks: management of acquisition database, indication of correct armband placement and real-time signal visualisation. Back-end, or communication part of the software was developed using myo-python library [15], that provides a Python wrapper for original MYO libraries. Signals from all the channels were visualized during the acquisition, and then stored to a database.

\section{B. Acquisition protocol}

During tests, the MYO armband was placed either on the dominant forearm or on the lower leg, depending on the test. While on the forearm, the armband was placed so that its distal side was approximately in the middle of ulna bone and its logo-block was aligned with virtual line passing through middle finger (see figure 2). On the lower leg, its distal side was set in the middle of tibia, with logo-block placed laterally to it, covering tibialis anterior muscle. In function of limb size

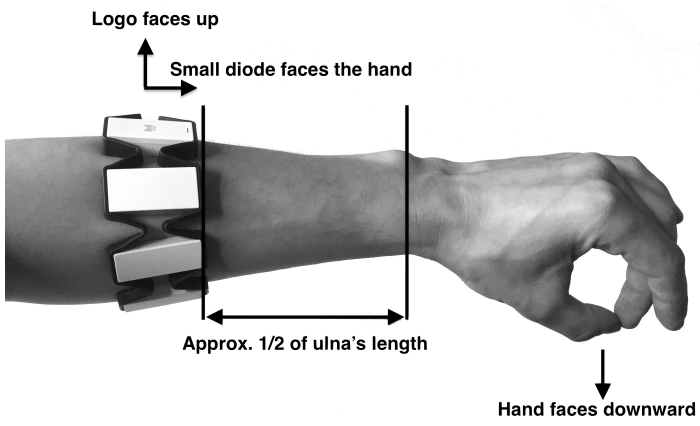

Fig. 2. Armband positioning on the forearm, example shown for finger tapping test.

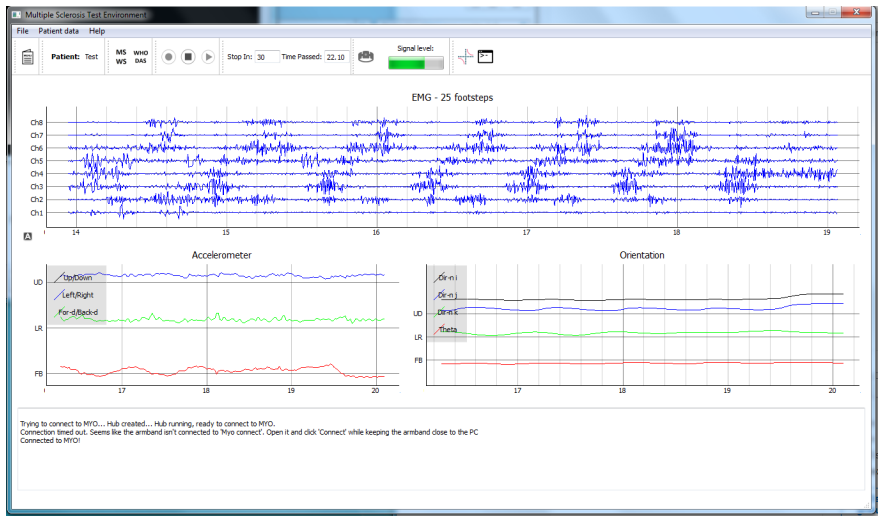

Fig. 3. The graphical user interface visualising acquired signals in real time.

armband was loosened or tightened using special clips to assert comfort of the subject and firm contact of EMG electrodes to the skin. Electrodes were evenly distributed around the limb.

The dataset for this publication was obtained during clinical follow-up of MS patients at Oslo University Hospital, Oslo, Norway. It consisted of 40 healthy controls and 30 MS patients. Tables I and II provide demographics and clinical evaluation statistics of the participants.

Each subject performed seven motor function tests: timed 25 foot walk (T25FW), finger tapping (FIT), finger-to-nose (FTN, foot tapping (FT), 9 hole peg test, hell-knee-shin test and Romberg's test. Within each test, two or three trials were performed, depending on subject's performance.

\section{Dataset}

Only the four following tests were considered in this study: T25FW, FIT, FTN and FT. Romberg's and heel-to-knee tests were rejected because of a low signal-noise ratio. In addition, 9 hole peg test was performed on two different equipments and thus was excluded from the study. Patients and healthy control (HC) formed two classes labelled by "1" and "0" respectively. Each of the four tests was used to form a separate dataset for a separate classification problem. Within the tests, each valid trial represented a sample. Later, for each person, a classification score obtained for all trials of a selected test were averaged to obtain a final score. 
TABLE I

DEMOGRAPHICS OF PARTICIPANTS

\begin{tabular}{|l|c|c|}
\hline Variable, mean (SD) & Patients, $\mathbf{~ = ~ 3 1 ~}$ & Healthy Controls, $\mathbf{~ = ~ 4 0 ~}$ \\
\hline Age & $37.7(7.4)$ & $33.3(6.8)$ \\
\hline Gender (\% of females) & 70 & 75 \\
\hline Height & $170.2(7.5)$ & $171.2(8.8)$ \\
\hline Weight & $71.4(16.4)$ & $67.1(12.4)$ \\
\hline Right handed (\%) & 86.7 & 90 \\
\hline
\end{tabular}

TABLE II

Clinical EVALUation of PatiEnts

\begin{tabular}{|l|c|}
\hline Variable, mean (SD, min-max) & Value, patients \\
\hline Age of first symptoms & $28.1(7.7,12-42)$ \\
\hline Age of MS onset & $32.5(7.5,19-49)$ \\
\hline Disease duration & $5.7(2.6,1.3-12.0)$ \\
\hline EDSS & $2.1(1.1,0.0-4.0)$ \\
\hline Total number of attacks & $2.3(1,1-5)$ \\
\hline
\end{tabular}

\section{Feature extraction}

Signals from all the trials were first visually analysed and regions of interest (ROIs) were manually extracted. Next, ROI lengths were equalised by trimming longer signals. Then, from each EMG channel, twelve temporal features were extracted [14]: number of zero crossings, waveform length (WL), slope sign change, Wilson amplitude (WA), root mean square, mean absolute value (MAV), integrated EMG (IEMG), signal variance and auto-regressive coefficients (4th order model).

Twelve spectral features were extracted as follows: the whole spectral band of the signal was partitioned into three sub-bands $(0-20 \mathrm{~Hz}, 20-50 \mathrm{~Hz}, 50-100 \mathrm{~Hz})$, within each band mean and median frequencies (MNF and MDF) were calculated, as well as energy (EN) and dispersion of spectrum. As for IMU, only accelerometer data was taken into account, extracted features were mean and median frequencies, without band partitioning.

\section{E. Dimensionality reduction}

Overall number of sample descriptors was 24 for each of eight EMG channels plus two features for each of three accelerometer channels, giving an overall dimensionality of 198 for each sample. In order prevent overfitting during classification, PCA was applied to the feature matrix of each test. Depending on the test, from six to ten principal components were kept. Features that were most represented in first principal components are EMG features: Wilson amplitude (WA), waveform length (WL) and energy (EN) in all three sub-bands (listed in table III).

TABLE III

FEATURES MOST REPRESENTED IN FIRST PRINCIPAL COMPONENTS

\begin{tabular}{|l|l|}
\hline \multicolumn{1}{|c|}{ Test } & \multicolumn{1}{c|}{ Features (EMG channels) } \\
\hline T25FW & WL(1), WA(1), EN $(8,1,5,2,4,6)$ \\
\hline FT & WL( $(4,5)$, WA $(4,5), \operatorname{EN}(2,7,5,1,8)$ \\
\hline \multirow{2}{*}{ FIT } & $\begin{array}{l}\text { WL }(2,1), \mathrm{WA}(1,2), \mathrm{EN}(8,7,5), \\
\text { MNF(0-20Hz) }(1,2,3,5,6)\end{array}$ \\
\hline \multirow{2}{*}{ FTN } & $\begin{array}{l}\text { WA }(3,5,6,4), \mathrm{WL}(3,5,6,4), \mathrm{EN}(1,6), \\
\text { IEMG }(2,4,5), \mathrm{MAV}(2,4,5)\end{array}$ \\
\hline
\end{tabular}

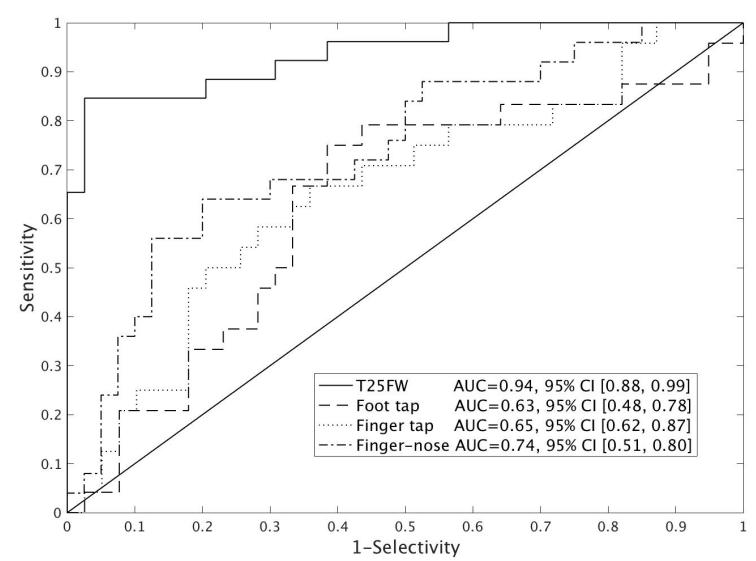

Fig. 4. ROC curves for four motor function tests, T25FW stands for timed 25 foot walk.

TABLE IV

CONFUSION MATRIX FOR COMBINED CLASSIFICATION APPROACH

\begin{tabular}{|c|c|c|}
\hline & Healthy controls & Multiple Sclerosis \\
\hline Healthy controls & 30 & 10 \\
\hline Multiple Sclerosis & 2 & 25 \\
\hline
\end{tabular}

\section{F. Classification}

Support vector machine (SVM) models were learned from four datasets corresponding to each of the tests, using svmlib library [16]. Polynomial kernel was used, best values of kernel and of regularization parameter were found using grid-search. We performed 5-fold cross validation of the result. Absolute value of SVM output is distance between the sample and separating hyperplane, while its sign represents estimated class label.

\section{RESULTS AND DISCUSSION}

For each data sample (single test trial), SVM model output characterises the sample score, probability of being either healthy or having MS. For each subject, scores corresponding to multiple trials of the same test were averaged. Then, sigmoidal function was applied to map the average scores onto the $[0,1]$ interval to then trace ROC curves for each of four tests 4 . Best performance obtained was for T25FW (area under ROC 95\% CI [0.88, 0.99]).

The obtained scores may be interpreted in different ways, depending on the classification strategy. Therefore, in situations where one single decision (for example, "no MS"/"possible MS") must be provided, results obtained from different tests should be combined. As an example of possible approach, we propose a rule that consists of diagnosing a subject to have MS if 1) his most positive score is larger, by absolute value, than his scores for other tests; or 2) if he has at least two positive scores. Confusion matrix for that approach reveals its high sensitivity (see table IV). 


\section{CONCLUSION AND FUTURE WORK}

In this study we assessed the classification capabilities of MYO armband and typical EMG processing pipeline, applied to a sample of MS patients and healthy subject. Timed 25 feet walk test has shown a superior performance (in terms of the area under ROC curve) compared to other tests. Possible explanation is the fact that walking requires coordination of the whole body and thus may be affected by MS-caused abnormalities in any body part. Other tests involve independent movement of either upper or lower limb and thus may not represent the actual state of a subject if his/her MS manifests elsewhere.

Possible development of this study have to consider, first of all, the acquisition protocol. In order to compare the data acquired by two different groups, a common protocol should be established, including subject's position and trajectory of walking. Also, special care should be taken considering the intra-class variability of the EMG signals caused by different skin and body fat conditions. Necessity of EMG normalisation with respect to maximum voluntary contraction level should be investigated. No common diagnostic protocol involving MYO armband may be established until these questions are assessed. Finally, as possible follow-up based on this study we are investigating the possibility of predicting MS disability based on regression of SVM scores on EDSS (Expanded Disability Status Scale) [17]. For that purpose, another dataset consisting only of MS patients with different levels of disability based on EDSS is currently being acquired.

\section{REFERENCES}

[1] W. I. McDonald, A. Compston, G. Edan, D. Goodkin, H. P. Hartung, F. D. Lublin, H. F. McFarland, D. W. Paty, C. H. Polman, S. C. Reingold, M. Sandberg-Wollheim, W. Sibley, A. Thompson, S. van den Noort, B. Y. Weinshenker, and J. S. Wolinsky, "Recommended diagnostic criteria for multiple sclerosis: Guidelines from the International Panel on the diagnosis of multiple sclerosis," Annals of Neurology, vol. 50 no. 1, pp. 121-127, Jul. 2001.

[2] C. H. Polman, S. C. Reingold, B. Banwell, M. Clanet, J. A. Cohen, M. Filippi, K. Fujihara, E. Havrdova, M. Hutchinson, L. Kappos, F. D. Lublin, X. Montalban, P. O'Connor, M. Sandberg-Wollheim, A. J. Thompson, E. Waubant, B. Weinshenker, and J. S. Wolinsky, "Diagnostic criteria for multiple sclerosis: 2010 Revisions to the McDonald criteria," Annals of Neurology, vol. 69, no. 2, pp. 292-302, Feb. 2011.

[3] M. Kaufman, D. Moyer, and J. Norton, "The significant change for the Timed 25-Foot Walk in the Multiple Sclerosis Functional Composite," Multiple Sclerosis Journal, vol. 6, no. 4, pp. 286-290, Aug. 2000.

[4] "9-Hole Peg Test (9-HPT)," http://www.nationalmssociety.org/ForProfessionals/Researchers/Resources-for-Researchers/Clinical-StudyMeasures/9-Hole-Peg-Test-(9-HPT).

[5] S. H. Alusi, J. Worthington, S. Glickman, and P. G. Bain, "A study of tremor in multiple sclerosis," Brain, vol. 124, no. 4, pp. 720-730, Apr. 2001.

[6] I. Jonkers, G. Nuyens, J. Seghers, M. Nuttin, and A. Spaepen, "Muscular effort in multiple sclerosis patients during powered wheelchair manoeuvres," Clinical Biomechanics (Bristol, Avon), vol. 19, no. 9, pp. 929-938, Nov. 2004.

[7] S.-S. Ayache, T. Al-ani, W.-H. Farhat, H.-G. Zouari, A. Créange, and J.-P. Lefaucheur, "Analysis of tremor in multiple sclerosis using HilbertHuang Transform," Neurophysiologie Clinique = Clinical Neurophysiology, vol. 45, no. 6, pp. 475-484, Dec. 2015.

[8] K. J. Kelleher, W. Spence, S. Solomonidis, and D. Apatsidis, "The characterisation of gait patterns of people with multiple sclerosis," Disability and Rehabilitation, vol. 32, no. 15, pp. 1242-1250, Jan. 2010.
[9] "Thalmic Labs - Makers of the Myo Gesture Control Armband," https://www.thalmic.com/.

[10] J. G. Abreu, J. M. Teixeira, L. S. Figueiredo, and V. Teichrieb, "Evaluating Sign Language Recognition Using the Myo Armband." IEEE, Jun. 2016, pp. 64-70.

[11] K. Akhmadeev, E. Rampone, T. Yu, Y. Aoustin, and E. Le Carpentier, "A testing system for a real-time gesture classification using surface EMG," IFAC-PapersOnLine, vol. 50, no. 1, pp. 11498-11 503, 2017.

[12] A. Atasoy, E. Kaya, E. Toptas, S. Kuchimov, E. Kaplanoglu, and M. Ozkan, "24 DOF EMG controlled hybrid actuated prosthetic hand," in Engineering in Medicine and Biology Society (EMBC), 2016 IEEE 38th Annual International Conference of The. IEEE, 2016, pp. 50595062.

[13] A. Phinyomark, F. Quaine, S. Charbonnier, C. Serviere, F. TarpinBernard, and Y. Laurillau, "EMG feature evaluation for improving myoelectric pattern recognition robustness," Expert Systems with Applications, vol. 40, no. 12, pp. 4832-4840, Sep. 2013.

[14] M. Hakonen, H. Piitulainen, and A. Visala, "Current state of digital signal processing in myoelectric interfaces and related applications," Biomedical Signal Processing and Control, vol. 18, pp. 334-359, Apr. 2015.

[15] NiklasRosenstein, "Myo-python: Python bindings for the Myo SDK," Jan. 2018.

[16] C.-C. Chang and C.-J. Lin, "LIBSVM: A library for support vector machines," ACM transactions on intelligent systems and technology (TIST), vol. 2, no. 3, p. 27, 2011.

[17] J. F. Kurtzke, "Rating neurologic impairment in multiple sclerosis: An expanded disability status scale (EDSS)," Neurology, vol. 33, no. 11, pp. 1444-1444, 1983. 